\title{
Canadian Public Health Laboratory Network Best Practices for COVID-19
}

\author{
Respiratory Virus Infections Working Group ${ }^{1}$
}

\begin{abstract}
The ability to detect severe acute respiratory syndrome coronavirus 2 (SARS-CoV-2), the causative agent of COVID-19, is a foundational component of Canada's containment and mitigation strategies. Laboratory confirmation of COVID-19 cases allows the appropriate clinical management and public health interventions. Whether the local goal is containment or mitigation will depend on local epidemiology of the pandemic. The Respiratory Virus Infections Working Group of the Canadian Public Health Laboratory Network has developed comprehensive Best Practice Guidelines for detection of SARS-CoV-2. Best practices for specimen collection, transportation, testing and biosafety are addressed from the perspective of Canadian public health laboratories to ensure a consistent approach across the country:

1. Population-based testing for COVID-19 should initially be carried out for surveillance

2. Nasopharyngeal swab is the specimen of choice for routine testing

3. Nucleic acid amplification tests (such as real-time reverse transcription polymerase chain reaction) are the method of choice for routine testing of SARS-CoV-2

4. The decentralization of nucleic acid amplification testing for COVID-19 to hospital or other high complexity medical laboratories should be promoted to increase test capacity and meet increased demands

5. In the early stages of the pandemic, positive (approximately 10-20) and negative (approximately 50 ) tests by a provincial laboratory require confirmation at the National Microbiology Laboratory

6. Co-circulation of other viral agents associated with influenza-like Illnesses (e.g. influenza A and $B$ and respiratory syncytial virus) should be monitored as capacity permits, as part of ongoing surveillance

7. Once validated, serological testing may be utilized for assessing the presence/absence of immune response to the SARS-CoV-2 at either the population or individual level for select indications, but is likely to be of limited utility in diagnosis of acute COVID-19 illness
\end{abstract}

These recommendations will be updated as new information becomes available.
This work is licensed under a Creative Commons Attribution 4.0 International License.

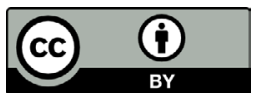

Affiliation

${ }^{1}$ Canadian Public Health Laboratory Network, Winnipeg, MB

*Correspondence: dionne.marcino@canada.ca

Suggested citation: Respiratory Virus Infections Working Group. Canadian Public Health Laboratory Network Best Practices for COVID-19. Can Commun Dis Rep 2020;46(5):113-8. http://doi.org/10.14745/ccdr.v46i05a02

Keywords: COVID-19, best practices, Canada, testing, surveillance

\section{Introduction}

Since the report of the novel coronavirus disease 2019 (COVID-19), caused by SARS-CoV-2 in late December 2019 in Wuhan, Hubei Province of China, the vast majority of countries have now reported laboratory-confirmed cases of COVID-19. Due to the continued spread of COVID-19, the situation was declared a pandemic by the World Health Organization on March 11, 2020 (1).

The clinical presentation of COVID-19, which is caused by SARS-CoV-2, is non-specific and overlaps with other seasonal respiratory viruses, including influenza. The ability to detect
SARS-CoV-2 in patients is critical for surveillance, diagnosis and clinical management of persons presenting with acute respiratory illness (ARI), influenza-like illness (ILI) and severe respiratory illness to support Canada's containment and mitigation strategies.

The purpose of SARS-CoV-2 testing can fall into two broad categories, and will depend on the local epidemiology and goals of public health strategies (containment vs. mitigation): 
1) Testing for the purpose of high probability case finding among persons presenting with ARI and ILI and appropriate exposure criteria is critical to ensure COVID-19 cases are identified in a timely fashion to ensure appropriate clinical and public health management can occur during the containment phase of the pandemic. In addition, when local numbers are low, testing will likely support aggressive case-finding strategies for early contact tracing and implementation of self-isolation. Once the virus becomes widespread, testing of community samples should be reserved for community-based surveillance programs, with the remainder of testing focused on hospitalized patients with ARI and those with risk factors for severe disease where the results of the test may influence decisions regarding care and treatment, infection control (including outbreaks), management of close contacts, and to support remote communities. It is important that the above management and prevention decisions should not be delayed pending testing results. Cases of COVID-19 have had co-infections with other viruses including influenza. Testing for influenza should continue for hospitalized patients to help support patient management with antivirals.

2) Population-based surveillance should occur for ongoing identification of COVID-19 cases and facilitate tracking of other common viral agents, such as respiratory syncytial virus, parainfluenza, adenovirus and rhinoviruses, that co-circulate during the influenza season and during other times of the year.

This Best Practices guidance should be used in conjunction with relevant provincial and territorial guidelines. The Public Health Agency of Canada will be posting regular updates and related documents (2).

\section{Surveillance}

Population-based surveillance is important during different stages of the COVID-19 pandemic. The areas of focus of surveillance will shift as testing priorities are realigned when the health system moves from a containment to mitigation phase. It is important to note that the existing technologies for COVID-19 detection are not sufficient in their performance to be applied as a general population screening tool, and targeted use of testing in populations where pretest probability is highest, or where potential benefit remains highest, remains an important principle of sample selection.

During containment, population-based surveillance is very important, as mildly symptomatic SARS-CoV-2 infection may play some role in community transmission. At this stage, the majority of patients tested for SARS-CoV-2 are ambulatory, with few hospitalized patients meeting exposure criteria to be a suspect case (3). During this time, it is important to conduct surveillance testing on a subset of hospitalized persons, and persons seen in ambulatory settings with ARI/ILI but no specific risk factors for COVID-19. Additional community surveillance should occur at long-term care homes, where the elderly patient population, often with comorbidities, are at greatest risk for complications and fatal infection. This surveillance could occur by testing an appropriate selection (as guided by outbreak control authorities) or all respiratory outbreak samples for SARS-CoV-2.

During mitigation, it is presumed that there will be widespread circulation of the virus throughout different sectors of the community. During this time, COVID-19 testing will shift to identifying cases among hospitalized patients, who represent the more severely ill. Community testing for SARS-CoV-2 will be less routinely available for ambulatory patients, though should be continued for ambulatory healthcare workers with ILI (and possibly ARI), institutional outbreaks, remote and confined/ congregate communities, and may be provided to populations with risk factors for severe disease (e.g. age 60 years or older, presence of comorbidities). Specific screening, sampling, specimen collection and testing guidelines will be developed by the local provincial healthcare system. Ambulatory surveillance programs should continue during a mitigation phase in order to provide some data on community prevalence of SARS-CoV-2, as this will support tracking the progress of the pandemic.

To assist with maximizing use of laboratory testing data to enable COVID-19 surveillance, hospitals or other high-complexity laboratories doing testing should contribute summary testing data to complement the data from testing at their provincial public health laboratory. These data can then help inform a local, provincial and federal snapshot of pandemic activity. Provinces should seek to perform adequate surveillance and case-finding test volumes, which will provide approximately a daily snapshot of disease prevalence in their test jurisdictions. The determination of that minimum volume is based on a number of factors and should be determined in cooperation with biostatistical or epidemiogical support.

Surveillance should also be in place to help with the global monitoring of the molecular epidemiology of SARS-CoV-2. This will help establish any geographic differences in strains circulating, and possible clinically relevant genomic variants. Molecular surveillance will also provide data to assist with monitoring for any diagnostic assay primer or probe mismatches to SARS-CoV-2 that might affect the performance characteristics of diagnostic assays. Such efforts should be coordinated across all jurisdictions, and led by World Health Organization-connected facilities such as the National Microbiology Laboratory (NML) in Winnipeg. While further research is necessary, it may inform questions of postinfection immunity and potential for reinfection, as well as assist with vaccine planning and design. While there currently is no specific antiviral therapy for SARS-CoV-2, genomic sequence data may be helpful in predicting resistant phenotypes if effective antivirals are developed.

Seroprevalence studies may also be conducted to assist with documenting the population attack rates from COVID-19 during the pandemic. These would be conducted by performing SARS-CoV-2 serology on a representative set of residual sera 
from across all age groups, and repeating this at set intervals over the coming months. The main challenge to conducting this activity is that no commercial assay has been validated for clinical testing at this time, although efforts for validation are underway in Canada, and the utility of such assays on a broad population scale is not yet affirmed.

\section{Diagnostic testing}

During the containment period, efforts will be directed at intense case finding to ensure early identification, early isolation, early diagnosis and early treatment as well as appropriate contact management and follow up. This will include both outpatient (ambulatory) and inpatient settings. Once the epidemiology of the outbreak suggests that containment is not feasible and resources will become strained, the laboratory will support the goal of mitigation and prioritize testing to the following groups of patients: 1) hospitalized patients with all degrees of ARI, including severe respiratory illness and ILI and milder respiratory illness; 2) patients for whom diagnostic testing will assist decisions regarding care, infection control (including outbreaks), or management of close contacts; 3 ) persons who died of an acute illness in which influenza or another respiratory virus such as SARS-CoV-2 is suspected; 4) healthcare workers with ARI/ILI; and 5) persons living in remote and isolated communities.

In the mitigation phase, when viral circulation in the community is established, testing may only occasionally be performed on outpatients; specific testing algorithms will be decided on by each provincial health system, with a likely focus similar to what is outlined above. Testing is not indicated for clinical management of persons with uncomplicated respiratory infection residing in communities where SARS-CoV-2 is circulating.

\section{Specimen type and collection}

The World Health Organization recently reported that SARS-CoV-2 has been detected in respiratory, fecal and blood specimens (4). Preliminary data report virus detection in upper respiratory samples $1-2$ days before symptom onset, which persists for 7-12 days in moderate cases and up to two weeks in severe cases. Virus has been cultured from respiratory tract samples up to eight days following symptom onset. Although SARS-COV-2 virus has also been detected in saliva, its use for diagnostic testing requires further investigations.

Viral ribonucleic acid (RNA) has been detected in feces in up to $30 \%$ of patients commencing day 5 after symptom onset, and this continues for up to five weeks in moderate cases. However, it is not clear whether this reflects shedding of infectious virus. While live virus has been cultured from stool in some cases, the role of fecal-oral transmission is not yet well understood.
At this time the focus of testing is on respiratory samples. Early data suggest that lower viral loads can be detected in nasopharyngeal swabs than in throat swabs (5), and as such they are the preferred upper respiratory tract specimen. In addition, they are also the preferred specimen for influenza detection, which can have a similar clinical presentation. Sputum is a useful lower respiratory tract specimen, and can be collected from patients with a productive cough. However, sputum induction is not recommended due to the risk of generating aerosols. Flocked swabs are recommended to collection of nasopharyngeal or nasal/throat specimens.

\section{Alternative collection devices}

In the event of a supply chain interruption and an inability to obtain flocked swabs or viral transport media, alternative options such as rayon on plastic or wires can be considered. Consideration to alternatives to viral transport media include phosphate buffered saline or alcohol for stabilization. Wooden swabs are considered inhibitory to nucleic acid-based testing, and therefore unless validated to the contrary, are not recommended. Any alternative specimen collection devices or transport media will require validation for use in clinical testing. Further information on alternative collection kits is available from U.S. Food and Drug Administration (6).

\section{Specimen pooling}

Pooling multiple specimens may be considered as a means of increasing throughput during periods of high submissions, and to preserve reagents during times of shortages. If the pool is positive, then each individual specimen within the pool must be retested to determine which specimen is positive. There is a trade-off of decreased sensitivity when specimens are pooled. Any laboratory considering pooling should do their own evaluation of the impact on sensitivity as this will be assay and laboratory specific, and use this to decide on the optimal number to pool in their setting. Work with influenza outbreaks has shown that sensitivity significantly drops if pooling more than four specimens. Laboratories may choose to run only noncritical specimens through a pooling protocol and preserve single specimen testing for patients with more severe illness (e.g. hospitalized patients). As percent positivity increases, the number of specimens within the pool for this to be efficient will need to be reduced; in general, once the test positive rate reaches the $8 \%-10 \%$ range, there is no benefit to pooling any number of specimens (Table 1).

\section{Specimen transport}

Specimens should be transported to the laboratory as soon as possible, preferably within 72 hours, on ice packs. If a longer delay is anticipated, specimens should be frozen at $-70^{\circ} \mathrm{C}$ or colder, and transported on dry ice. However, specimens should not be frozen at $-20^{\circ} \mathrm{C}$, as this may affect the recovery of the virus if culture is required. If $-70^{\circ} \mathrm{C}$ or below/dry ice is not available, specimens should remain at $4^{\circ} \mathrm{C}$ and be shipped as 
Table 1: Preferred and alternative specimen types

\begin{tabular}{|c|c|c|}
\hline $\begin{array}{l}\text { Nature of } \\
\text { illness }\end{array}$ & Specimen of choice & $\begin{array}{c}\text { Alternative } \\
\text { specimens }\end{array}$ \\
\hline $\begin{array}{l}\text { Mild/ } \\
\text { moderate } \\
\text { influenza-like } \\
\text { illness }\end{array}$ & $\begin{array}{l}\text { Nasopharyngeal swab } \\
\text { Video demonstration of } \\
\text { nasopharyngeal swab } \\
\text { collection can be accessed } \\
\text { at http://www.youtube.com/ } \\
\text { watch?v=TFwSefezlHU }\end{array}$ & $\begin{array}{l}\text { Deep nasal swab, } \\
\text { throat swab or } \\
\text { both https://vimeo. } \\
\text { com/397169241 }\end{array}$ \\
\hline $\begin{array}{l}\text { Severe } \\
\text { respiratory } \\
\text { illness }\end{array}$ & $\begin{array}{l}\text { Nasopharyngeal swab } \\
\text { AND endotracheal or } \\
\text { bronchoalveolar lavage. Sputum } \\
\text { (if productive cough) }\end{array}$ & $\begin{array}{l}\text { Sputum, throat } \\
\text { swab }\end{array}$ \\
\hline Autopsy & $\begin{array}{l}\text { Nasopharyngeal swab AND } \\
\text { throat swab } \\
\text { Lung tissue or other tissues } \\
\text { from suspected organ } \\
\text { involvement. Specimens should } \\
\text { be fresh or frozen at }-70^{\circ} \mathrm{C} \text { or } \\
\text { below. Do not put into formalin } \\
\text { fixative }\end{array}$ & Not applicable \\
\hline
\end{tabular}

soon as possible. Specimens should be transported as Transport of Dangerous Goods-defined diagnostic specimens per the usual practice for seasonal influenza specimens, and no enhanced precautions are necessary. See the PHAC SARS-CoV-2 Biosafety Advisory for more information (7).

Specimen tubes should be appropriately labelled and requisition correctly and fully completed, with matching patient names and unique identifiers, and relevant clinical and/or public health required information.

\section{Testing methods}

While other methods exists for the detection of SARS-CoV-2, detection methods in clinical laboratories are limited to molecular detection using nucleic acid amplification tests (NAAT) and viral culture.

\section{Nucleic acid amplification tests}

At the time of this publication, there are an increasing number of commercial assays available for detection of SARS-CoV-2. Many laboratories are implementing in-house, laboratory-developed tests based on the detection of the RNA-dependent RNA polymerase, envelope and nucleocapsid genes, while others are implementing commercial assays that detect a variety of viral targets. Some laboratories have a pan-beta coronavirus RNA polymerase NAAT, which is then confirmed by nucleic acid sequencing, although most laboratories have moved to real-time methods that directly identify two different genetic targetsgene sequencing is reserved for cases where a single target is indeterminate on the real-time reverse transcription polymerase chain reaction (rRT-PCR) assay and further clarification of the laboratory result is clinically indicated.
As a result of the evolution of the outbreak into a pandemic, and SARS-CoV-2 no longer being a rare laboratory test finding, detection of a single target under well-validated conditions is sufficient for laboratory confirmation of SARS-CoV-2.

Although little data exist on the diagnostic performance of current NAAT tests, based on preliminary data from Canadian laboratories the level of detection tests have excellent analytical sensitivity ( $95 \%$ limit of detection below 10 copies per reaction) and specificity. During level of detection tests validation, laboratories should determine the maximum cycle threshold value for target detection, using the $95 \%$ limit of detection generated in their laboratory as a guide. They should also decide whether an indeterminate cycle threshold range for that particular assay is required, and what cycle threshold values to include in the indeterminate range. Patients who initially test negative should be retested if the clinical suspicion of COVID-19 remains high, in particular among hospitalized patients who are not clinically improving. Lower respiratory tract samples should be obtained from patients with evidence of pneumonia to increase clinical sensitivity. Test performance among patients with different severities of illness (e.g. asymptomatic, mild illness, hospitalized) is likely to differ, and these differences have not been well characterized. Routine testing of asymptomatic patients is not recommended. Ongoing evaluation of commercially available tests, as they are developed, will be important to characterize their performance in the clinical setting and throughout the pandemic. Public health laboratories should take appropriate initiatives and help establish additional testing sites in their respective jurisdictions.

\section{Point-of-care molecular testing}

Commercial molecular detection assays are, and more will become, licensed by Health Canada for point-of-care (POC) use outside the laboratory. Before facilities in Canada consider using any POC or a non-class III device "off label" for near POC testing, an implementation and quality plan should be made with a clinical or medical microbiologist and an appropriate laboratory medical director. Where possible, a provincial system should be set up for capturing the data generated from POC testing to assist with laboratory surveillance. As with any medical laboratory activities, adherence to any appropriate personal health information, medical laboratory accreditation and medical laboratory licensure regulations and standards must be considered in advance of offering such testing.

\section{Virus isolation}

Virus isolation is limited to laboratories that have licensed containment level (CL) 3 capabilities, and will not play a major role in the diagnosis of COVID-19 patients. It will mainly be used to propagate virus for the generation of positive RNA control material required for NAATs. It may also be required to support growth-based serological assays if developed (e.g. microneutralization), vaccine development, and other areas of research. 


\section{Serology}

Methods for serologic diagnosis are being developed but have not yet been introduced into routine clinical use in Canada or other countries. Several platforms targeting various immunoglobulins (lgM, lgG, $\lg A$ ) and total antibodies against different SARS-CoV-2 antigens, such as spike protein and nucleocapsid protein, are available for evaluation. Based on available literature, detection of serological response appears to be less reliable in the first week post-symptom onset where sensitivity is low. The sensitivity of detection increases by 14 days post-symptoms onset. Duration of seropositivity postinfection and whether the immune response offers or correlates with protection from reinfection needs to be determined before interpretation relating to immunity can be made.

The role of serology in diagnosis of acute illness and patient management is likely to be of limited utility. Once the dynamics of serological response are better understood, serology may have a role in the following: use in seroepidemiology studies to better understand the proportion undiagnosed in the population over time and provide a more accurate estimate of attack rate; an adjunct to rRT-PCR for diagnostic testing in patients who are rRTP-CR negative, late in the course of their illness, and have significant contact management challenges that would be well-informed by supportive serology; to implement control measures and to effectively manage significantly at-risk populations, including assessing them for serostatus; and once a vaccine is available it may be used to determine, among high-risk populations, who should be prioritized for earlier vaccination.

Two testing modalities are currently available commercially, enzyme-linked immunosorbent assay- (ELISA-) based assays and POC assays. The performance characteristics of both modalities need to be determined; in particular, sensitivity, specificity, positive predictive value and negative predictive value, in addition to the interpretation of positive results.

The ELISA-based methods are amenable to high-throughput processing, appropriate quality control and assurance, are less susceptible to operator subjectivity in interpretation and reporting of their results can be easily integrated into existing laboratory information systems. The ELISA methods are also capable of providing some quantitative estimate of how much antibody is present. They are, however, more labor-intensive, require special equipment, reagents and laboratory expertise and do not provide rapid results. As an estimate of protection of the immune response, ELISA results should be compared with results of virus neutralization assays. However, at present, neutralization assays are not produced commercially and can only be employed in high complexity laboratories capable of tissue and viral culture, limiting their widespread use.

Most POC tests are immunochromatographic and lateral-flow based and as a result, provide easy to read results in as little as 30 minutes without the requirement of extensive training or specialized equipment. They are particularly beneficial for use in remote areas with limited access to centralized laboratorybased testing and/or limited local laboratory infrastructure. The same guidelines outlined above for POC molecular assays apply to $\mathrm{POC}$ serology assays. Use under such conditions requires particular attention and effort to ensure quality control and assurance, such as participation in external quality assessment, to maintain high-quality testing. Similarly, provisions for maintaining appropriate data and quality records of $\mathrm{POC}$ test results are necessary before their implementation into routine use.

\section{External quality assurance}

Any laboratory implementing testing for SARS-CoV-2 should do so according to the medical laboratory regulations in place in their jurisdiction. As is required for other microbiology clinical tests, they must be enrolled in available external quality assessment programs that can be accessed provincially, nationally and/or internationally. This is particularly important when providing testing for an emerging pathogen such as SARS-CoV-2. The development and provision of standardized serology panels to support implementation and proficiency testing will be key to the successful implementation of serology assays in Canadian laboratories.

\section{Detection of other respiratory viruses}

The emergence of COVID-19 comes at a time when many regions in the Northern Hemisphere are experiencing their respiratory virus season and there are data to suggest that co-infections can occur; however, the clinical implications of co-infection on patient outcomes are not clear. It is expected that with wide spread circulation of the virus, the diagnostic capacities of laboratories may be exceeded and will require the suspension of some services or the use of contingency plans thus making it unrealistic to expect broad routine testing for the other viruses. However, the detection of influenza, particularly in patients requiring hospitalization or those with comorbidities putting them at risk for complications, should continue to help guide patient management with anti-influenza agents.

\section{Biosafety considerations}

The SARS-CoV-2 is a risk group (RG) 3 pathogen. Propagation or culture of the virus is restricted to laboratories that have federally licensed CL3 facilities. The SARS-CoV-2 is transmitted from respiratory droplet spread and, as such, respiratory specimens would be considered potential sources of virus. Although there are limited data that suggest SARS-CoV-2 can be detected in blood and stool, there are no data at this time that suggest these are a source of infection. Non-propagative diagnostic activities using specimens that do not result in the 
concentration or extraction of the pathogen, such as routine chemistry, hematology or urinalysis can continue using standard precautions. Respiratory specimens from patients with suspect COVID-19 can be safely handled in CL2 facilities with additional precautions including the following: a lab coat, gloves, and eye protection are worn when handling primary specimens; centrifugation of primary specimens is carried out in sealed safety cups, or rotors, that are loaded/unloaded in a Class II biological safety cabinet (BSC) or other primary containment device; a certified Class II BSC, or other primary containment device, is used for procedures that may produce infectious aerosols including pipetting; and respiratory protection that provides a level of filtration of $95 \%$ or greater (e.g. N95) is worn where aerosol generating activities cannot be contained within a BSC or other primary containment device.

It is recommended that laboratories perform a local risk assessment on activities involving specimens from COVID-19 patients to determine if additional precautions are required.

Virus culture should not be conducted on respiratory specimens in a CL2 laboratory when a novel or emerging pathogen is suspected as they are RG 3 pathogens. Virus culture, if required, may be considered if the specimen has been tested for these pathogens and is negative by rRT-PCR.

\section{Disinfection}

Based on currently available evidence, chemical disinfectants that are effective against enveloped viruses are suitable for decontamination of SARS-CoV-2, provided they are used according to manufacturer's recommendations. Particular attention should also be given to the correct contact time (e.g. 10 minutes), dilution (i.e. concentration of the active ingredient) and expiry date of the working solution preparation. Such effective disinfectants include sodium hypochlorite (bleach), 70\% ethanol, $0.5 \%$ hydrogen peroxide, quaternary ammonium compounds and phenolic compounds. It is possible other biocidal agents may be less effective (e.g. 0.05\%-0.2\% benzalkonium chloride, $0.02 \%$ chlorhexidine digluconate).

Sodium hypochlorite (bleach) at a concentration of 1,000 ppm $(0.1 \%)$ is recommended for general surface disinfection and 10,000 ppm (1\%) for disinfection of blood spills.

See the PHAC SARS-CoV-2 Biosafety Advisory (7) and WHO Laboratory Biosafety Guidance Related to the Novel Coronavirus (2019-nCoV): Interim Guidance (4) for more information.

\section{Authors' statement}

The Respiratory Virus Infection (ReVI) Working Group of the Canadian Public Health Laboratory Network (CPHLN) is dedicated to providing leadership and guidance on topics related to respiratory viral pathogens, including laboratory response to emerging respiratory viruses. The ReVI Working Group is comprised of leaders from public health laboratories across Canada.

\section{Conflict of interest}

None.

\section{Acknowledgements}

The Respiratory Virus Infection Working Group would like to thank members of the Canadian Public Health Laboratory Network (CPHLN) Secretariat, S Radons Arneson and D Marcino, for coordination of document synthesis. We would also like to thank the Laboratory Directors Council of the CPHLN for review of the document.

\section{References}

1. Word Health Organization. Coronavirus disease 2019 (COVID-19) Situation Report 65 WHO; 2020. www.magnetmail. net/actions/email_web_version.cfm?ep=SUWFhCwRmEENOSZG C2yUcSbCyNDIzfxRuESmZzmC1hV6D6gsrpmRNviR2xWgwjflHN fEpisYU8RnPs2o4MIn4jwLPeBFAtFidmBq8s2Vxpyc3k6OYzIPbcs RDZOr_NsG

2. Public Health Agency of Canada. Coronavirus disease (COVID-19): For health professionals. Ottawa (ON): PHAC; modified April 24, 2020. https://www.canada.ca/en/ public-health/services/diseases/2019-novel-coronavirusinfection/health-professionals.html

3. Lin M, Beliavsky A, Katz K, Powis JE, Ng W, Williams V, Science M, Groves H, Muller MP, Vaisman A, Hota S, Johnstone J, Leis JA. What can early Canadian experience screening for COVID-19 teach us about how to prepare for a pandemic. CMAJ. $2020 \mathrm{Mar}$ 6. pii: cmaj.200305 [Epub ahead of print]. https://www.cmaj.ca/ content/cmaj/early/2020/03/06/cmaj.200305.full.pdf

4. World Health Organization. Laboratory biosafety guidance related to the novel coronavirus (2019-nCoV). Interim guidance. WHO; 12 February, 2020. https://www.who. int/docs/default-source/coronaviruse/laboratory-biosafet y-novel-coronavirus-version-1-1.pdf?sfvrsn=912a9847_2

5. Zou L, Ruan F, Huang $M$, Liang L, Huang $H$, Hong $Z$, Yu J, Kang M, Song Y, Xia J, Guo Q, Song T, He J, Yen HL, Peiris M, Wu J. SARS-CoV-2 Viral Load in Upper Respiratory Specimens of Infected Patients. N Engl J Med 2020 Mar;382(12):1177-9. [Epub ahead of print]. DOI PubMed

6. U.S. Food and Drug Administration. FAQs on Diagnostic Testing for SARS-CoV-2. https://www.fda.gov/medical-devices/ emergency-situations-medical-devices/faqs-diagnostic-testingsars-cov-2

7. Public Health Agency of Canada. SARS-CoV-2 (Severe acute respiratory syndrome-related coronavirus 2) Biosafety Advisory. Ottawa (ON): PHAC; February 29, 2020 https://www.canada. ca/en/public-health/services/laboratory-biosafety-biosecurity/ biosafety-directives-advisories-notifications/novel-coronavirusjanuary-27.html 